PROCEEDINGS OF THE

AMERICAN MATHEMATICAL SOCIETY

Volume 125, Number 11, November 1997, Pages 3321-3329

S 0002-9939(97)04095-1

\title{
CHEBYSHEV MEASURES
}

\author{
RAPHAËL CERF AND CARLO MARICONDA
}

(Communicated by J. Marshall Ash)

\begin{abstract}
We introduce Chebyshev measures. We generalize the representation theorem concerning both measures admitting a density function which is a $T$-system and oriented measures.
\end{abstract}

\section{INTRODUCTION}

We are concerned with the problem of representing the values of a vector measure through its restriction to a nice family of sets. This problem has been handled for a vector measure defined on an interval and admitting a continuous density function forming a Chebyshev system (a function $f:[0,1] \rightarrow \mathbb{R}^{n}$ is a $T$-system if $\operatorname{det}\left[f\left(x_{1}\right), \cdots, f\left(x_{n}\right)\right]>0$ when $\left.x_{1}<\cdots<x_{n}\right)([4],[5])$. In this case, to each interior point of the range correspond exactly two dual canonical finite unions of intervals. The proof relies on geometrical considerations and on the fact that a linear combination of the components of a $n$-dimensional vector function which is a $T$-system has at most $n$ zeroes. $T$-systems have been traditionally applied to approximation theory and to moment problems in statistics $([4],[5])$; here we consider them from the point of view of Measure Theory.

In dealing with the Lyapunov theorem on the range of vector measures and a bang-bang control problem ([1], [2]), we were led incidentally to prove a weaker version of the aforementioned theorem. We thank warmly Fabrice Gamboa for introducing us to the field of Chebyshev systems, especially because their relationship with the Lyapunov theorem does not appear at all in control theory literature. Our proofs differed strongly from the previous ones. In the case of continuous densities [2], they were based on the differentiability of the measure and on a global inversion argument. We generalized the result to unnecessarily absolutely continuous measures, but still with $n$ determinant conditions (oriented measures)[3] which allowed us to prove inductively the representation theorem with the help of an elementary topological argument.

The knowledge of the previous works on $T$-systems suggested to us that our result should hold with only one determinant condition. Here we introduce Chebyshev measures: they form a broad class of vector measures (unnecessarily defined on

Received by the editors August 29, 1995 and, in revised form, June 13, 1996.

1991 Mathematics Subject Classification. Primary 41A50, 46G10, 52A20.

Key words and phrases. Oriented measure, vector measure, Lyapunov, Chebyshev, T-system.

The second author (C.M.) was supported by a Grant of the Consiglio Nazionale delle Ricerche (Grant 203.01.62).

(C) 1997 American Mathematical Society 
an interval) admitting a representation property through canonical sets and whose range is strictly convex.

Our new argument is direct and uses the invariance domain theorem.

\section{General Framework}

Throughout the paper, we deal with the following objects:

- a measurable space $(X, \mathcal{A})$,

- a non-trivial positive measure $\nu$ defined on $\mathcal{A}$,

- a vector measure $\mu=\left(\mu_{1}, \cdots, \mu_{n}\right)$ defined on $\mathcal{A}$ with values in $\mathbb{R}^{n}$,

- an increasing family of measurable sets $\left(M_{i}\right)_{i \in[0,1]}$ such that $M_{0}=\emptyset, M_{1}=X$.

We suppose that the $M_{i}$ 's are distinct modulo $\nu$; i.e.

$$
\forall i, j \in[0,1] \quad i<j \quad \Longrightarrow \quad \nu\left(M_{i}\right)<\nu\left(M_{j}\right) .
$$

The total variation $|\mu|$ is the scalar measure $|\mu|=\left|\mu_{1}\right|+\cdots+\left|\mu_{n}\right|$ where the $\left|\mu_{i}\right|$ are the usual total variations of the scalar measures $\mu_{i}$. We make the following assumption.

Assumption. The measures $\nu$ and $\mu$ are non-atomic with respect to the family $\left(M_{i}\right)$; i.e.

$$
\begin{array}{rllll}
\forall E \in \mathcal{A} \quad \nu(E) \neq 0 & \Longrightarrow \quad \exists i \in[0,1] & & 0<\nu\left(E \cap M_{i}\right)<\nu(E), \\
\forall E \in \mathcal{A} \quad|\mu|(E) \neq 0 & \Longrightarrow \quad \exists i \in[0,1] & 0<|\mu|\left(E \cap M_{i}\right)<|\mu|(E) .
\end{array}
$$

Remark. This assumption guarantees that the maps $i \mapsto \nu\left(M_{i}\right)$ and $i \mapsto \mu\left(M_{i}\right)$ are continuous. Moreover the measures $\nu$ and $\mu$ are non-atomic.

Example 2.1 (linear intervals). This general framework stems from the case where $X$ is the interval $[0,1]$ of $\mathbb{R}, \mathcal{A}$ is the Lebesgue $\sigma$-field, $\nu$ is the Lebesgue measure, the $M_{i}$ 's are the intervals $[0, i]$ and $\mu$ is a non-atomic vector measure on $[0,1]$. In this situation, the previous assumption on $\mu$ turns out to be equivalent to the non-atomicity of $\mu$.

Example 2.2 (circular annulus). Let $X=B_{m}$ be the unit ball of $\mathbb{R}^{m}$ equipped with the Lebesgue measure $(\nu, \mathcal{A})$. We take $M_{i}$ to be the ball of radius $i$. Finally let $\mu$ be any vector measure which is absolutely continuous with respect to $\nu$. The assumption on $\mu$ is here equivalent to the fact that the spheres have a zero $|\mu|-$ measure.

\section{Chebyshev measures}

We denote by $\mathcal{S}_{n}$ the symmetric group of order $n$ and, for $\sigma$ in $\mathcal{S}_{n}$, by $\epsilon(\sigma)$ its sign. To the vector measure $\mu$ we associate a determinant measure $\operatorname{det} \mu$.

Definition 3.1 (determinant measure). The measure $\operatorname{det} \mu$ is the measure defined on the product space $\left(X^{n}, \mathcal{A}^{\otimes n}\right)$ by

$$
\operatorname{det} \mu=\sum_{\sigma \in \mathcal{S}_{n}} \epsilon(\sigma) \mu_{\sigma(1)} \otimes \cdots \otimes \mu_{\sigma(n)} .
$$

This is the only measure whose restrictions to the product sets $A_{1} \times \cdots \times A_{n}$ satisfy $\operatorname{det} \mu\left(A_{1} \times \cdots \times A_{n}\right)=\operatorname{det}\left[\mu\left(A_{1}\right), \cdots, \mu\left(A_{n}\right)\right]$. 
The definition of a Chebyshev measure will involve the following subset of $X^{n}$ :

$$
P=\bigcup_{0 \leq i_{1} \leq \cdots \leq i_{n-1} \leq 1} M_{i_{1}} \times\left(M_{i_{2}} \backslash M_{i_{1}}\right) \times \cdots \times\left(M_{i_{n-1}} \backslash M_{i_{n-2}}\right) \times\left(X \backslash M_{i_{n-1}}\right) .
$$

We assume that $P$ belongs to the product $\sigma$-field $\mathcal{A}^{\otimes n}$.

Examples. In the case of the linear intervals (Example 2.1) we have

$$
P=\left\{\left(x_{1}, \cdots, x_{n}\right) \in[0,1]^{n}: 0 \leq x_{1} \leq \cdots \leq x_{n} \leq 1\right\} .
$$

In the case of the circular annulus (Example 2.2) we have

$$
P=\left\{\left(x_{1}, \cdots, x_{n}\right) \in\left(B_{m}\right)^{n}: 0 \leq\left|x_{1}\right| \leq \cdots \leq\left|x_{n}\right| \leq 1\right\} .
$$

Definition 3.2 (Chebyshev measure). The vector measure $\mu$ is a $T_{\nu}$-measure if the measure $\operatorname{det} \mu$ satisfies

$$
\forall A \in \mathcal{A}^{\otimes n}, \quad A \subset P, \quad \nu^{\otimes n}(A)>0 \quad \Longrightarrow \quad \operatorname{det} \mu(A)>0 .
$$

The symbol $T_{\nu}$ stands for Chebyshev measure.

Remark. Any non-atomic positive scalar measure $\mu$ is a Chebyshev measure with respect to itself. In fact, Lyapunov's theorem yields the existence of an increasing family $\left(M_{i}\right)$ such that $\mu\left(M_{i}\right)=i \mu(X)$ for $i$ in $[0,1]$. Using the Hahn decomposition, any non-atomic scalar signed measure is the difference of two Chebyshev measures with respect to its total variation.

If $\mu$ is absolutely continuous with respect to $\nu$, this definition might be translated in terms of the density function.

Definition 3.3. Let $f=\left(f_{1}, \cdots, f_{n}\right)$ be a measurable vector-valued function defined on $X$. We say that $f=\left(f_{1}, \cdots, f_{n}\right)$ is a $T_{\nu}$-system if the determinant $\operatorname{det}\left[f\left(x_{1}\right), \cdots, f\left(x_{n}\right)\right]$ is positive for $\nu^{\otimes n}$-almost all $\left(x_{1}, \cdots, x_{n}\right)$ in $P$.

This definition is a slight generalization of the classical one which deals only with functions defined on an interval.

Theorem 3.4. Suppose $\mu$ is absolutely continuous with respect to $\nu$. Let $f=$ $\left(f_{1}, \cdots, f_{n}\right)$ be its density function. Then $\mu$ is a $T_{\nu}$-measure if and only if $f$ is a $T_{\nu}$-system.

Proof. Remark first that for any measurable set $A$ of $X^{n}$ we have

$$
\begin{aligned}
\operatorname{det} \mu(A) & =\int_{A} \sum_{\sigma \in \mathcal{S}_{n}} \epsilon(\sigma) f_{\sigma(1)}\left(x_{1}\right) \cdots f_{\sigma(n)}\left(x_{n}\right) d \nu^{\otimes n}\left(x_{1}, \cdots, x_{n}\right) \\
& =\int_{A} \operatorname{det}\left[f\left(x_{1}\right), \cdots, f\left(x_{n}\right)\right] d \nu^{\otimes n}\left(x_{1}, \cdots, x_{n}\right) .
\end{aligned}
$$

Suppose that $f$ is a $T_{\nu}$-system. Let $A$ be a measurable subset of $P$ of positive $\nu^{\otimes n}$ measure. The domain of integration has a positive measure and the integrand is positive $\nu^{\otimes n}$-almost everywhere on this domain. It follows that $\operatorname{det} \mu(A)$ is positive. Conversely, assume that $\mu$ is $T_{\nu}$-measure and set

$$
A=\left\{\left(x_{1}, \cdots, x_{n}\right) \in P: \operatorname{det}\left[f\left(x_{1}\right), \cdots, f\left(x_{n}\right)\right] \leq 0\right\} .
$$


Clearly $\int_{A} \operatorname{det}\left[f\left(x_{1}\right), \cdots, f\left(x_{n}\right)\right] d \nu^{\otimes n}\left(x_{1}, \cdots, x_{n}\right) \leq 0$. Assume that $\nu^{\otimes n}(A)>0$. By the very definition of a $T_{\nu}$-measure, we have $\operatorname{det} \mu(A)>0$. However the initial formula yields

$$
\operatorname{det} \mu(A)=\int_{A} \operatorname{det}\left[f\left(x_{1}\right), \cdots, f\left(x_{n}\right)\right] d \nu^{\otimes n}\left(x_{1}, \cdots, x_{n}\right) \leq 0,
$$

which is absurd. Thus $\nu^{\otimes n}(A)=0$.

There is a huge literature concerning Chebyshev systems of continuous functions defined on an interval. They were originally introduced in interpolation theory. Their general properties (in the case of continuous functions) have been thoroughly studied ([4], [5]).

Example 3.5 (circular annulus). Let $f=\left(f_{1}, \cdots, f_{n}\right):[0,1] \rightarrow \mathbb{R}^{n}$ be a $T$-system with respect to the Lebesgue measure on $[0,1]$ and the family of intervals $[0, i]$. Then the function $g(x)=f(|x|)$ defined on the unit ball $B_{m}$ is a $T$-system with respect to the elements defined in Example 2.2.

\section{Fundamental properties}

Notation. For a $k$-tuple of measurable sets $A_{1}, \cdots, A_{k}$ by $A_{1}<\cdots<A_{k}$ we mean that the $A_{i}$ 's are non negligible for $\nu$ and that there exist $i_{0}<\cdots<i_{k}$ such that $A_{1} \subset M_{i_{1}} \backslash M_{i_{0}}, \cdots, A_{k} \subset M_{i_{k}} \backslash M_{i_{k-1}}$.

The non-atomicity assumption on $\nu$ implies the following result.

Proposition 4.1. For each non-negligible set $E$ and for each integer $m$ there exist measurable sets $E_{1}, \cdots, E_{m}$ such that

$$
E=E_{1} \cup \cdots \cup E_{m} \quad \text { and } \quad E_{1}<\cdots<E_{m} .
$$

In particular $P$ is not $\nu^{\otimes n}-$ negligible.

Proof. Let $E$ be a set of positive $\nu$ measure. The map $i \in[0,1] \mapsto \nu\left(M_{i} \cap E\right)$ being continuous and increasing, there exist $0<i_{1}<\cdots<i_{m-1} \leq 1$ such that $\nu\left(M_{i_{l}} \cap E\right)=(l / m) \nu(E)$ for $l$ in $\{1, \cdots, m-1\}$. Then the sets $E_{l}=$ $\left(M_{i_{l}} \backslash M_{i_{l-1}}\right) \cap E$ for $l \in\{1, \cdots, m-1\}, E_{m}=E \backslash M_{i_{m-1}}$, satisfy the required conditions. Applying this result to $E=X$ and $m=n$, we obtain a subset of $P$ of positive $\nu^{\otimes n}$ measure.

If $\rho$ is a measurable function on $X$, its support is the set supp $\rho=\{x: \rho(x) \neq 0\}$. If $\rho$ belongs to $L_{\mu}^{1}(X)$, by $\mu(\rho)$ we denote the column vector

$$
\mu(\rho)=\int_{X} \rho d \mu=\left(\int_{X}^{\mathrm{t}} \rho d \mu_{1}, \cdots, \int_{X} \rho d \mu_{n}\right) .
$$

A direct consequence of the definitions is that if $A_{1}<\cdots<A_{n}$ then the determinant $\operatorname{det}\left[\mu\left(A_{1}\right), \cdots, \mu\left(A_{n}\right)\right]$ is positive. A more important fact concerning $T_{\nu}$-measures is that this characteristic property carries on from sets to positive functions.

Theorem 4.2. Suppose $\mu$ is a $T_{\nu}$-measure. If $\rho_{1}, \cdots, \rho_{n}$ are $n \mu$-integrable nonnegative functions such that supp $\rho_{1}<\cdots<$ supp $\rho_{n}$ then the determinant $\operatorname{det}\left[\mu\left(\rho_{1}\right), \cdots, \mu\left(\rho_{n}\right)\right]$ is positive.

Let us first state a preparatory lemma. 
Lemma 4.3. Let $\rho_{1}, \cdots, \rho_{n}$ be $n \mu$-integrable functions. Then

$$
\operatorname{det}\left[\int_{X} \rho_{1} d \mu, \cdots, \int_{X} \rho_{n} d \mu\right]=\int_{X} \cdots \int_{X} \rho_{1}\left(s_{1}\right) \cdots \rho_{n}\left(s_{n}\right) \operatorname{det} \mu\left(s_{1}, \cdots, s_{n}\right) .
$$

Proof of the lemma. The identity is obviously true whenever $\rho_{1}, \cdots, \rho_{n}$ are characteristic functions. The monotone class theorem yields the result. Another scheme of proof is to develop the determinant and to transform each product of integrals into an $n$-dimensional integral with respect to a judicious product measure.

Proof of Theorem 4.2. We apply the lemma. The domain of integration of the $n$-fold integral is reduced to supp $\rho_{1} \times \cdots \times \operatorname{supp} \rho_{n}$ on which the determinant measure $\operatorname{det} \mu$ is positive (by the definition of a $T_{\nu}$-measure). Hence the $n$-fold integral is positive.

Corollary 4.4. Suppose $\mu$ is a $T_{\nu}$-measure and let $\rho_{1}, \cdots, \rho_{p}$ be $p$ non-negative $\mu$-integrable functions such that supp $\rho_{1}<\cdots<$ supp $\rho_{p}$. If

$$
\lambda_{1} \int_{X} \rho_{1} d \mu+\cdots+\lambda_{p} \int_{X} \rho_{p} d \mu=0
$$

for some $\left(\lambda_{1}, \cdots, \lambda_{p}\right) \neq(0, \cdots, 0)$, then $p$ is strictly greater than $n$.

Proof. If $p=n$ Theorem 4.2 yields $\operatorname{det}\left[\mu\left(\rho_{1}\right), \cdots, \mu\left(\rho_{n}\right)\right] \neq 0$, a contradiction. If $p<n$, by Proposition 4.1, we can decompose supp $\rho_{1}$ into the union of $n-p+1$ non-negligible sets $A_{i}, 1 \leq i \leq n-p+1$. Then if we set

$$
\left(\tilde{\lambda}_{k}, \tilde{\rho}_{k}\right)= \begin{cases}\left(\lambda_{1}, \rho_{1} \chi_{A_{k}}\right) & \text { if } 1 \leq k \leq n-p+1 \\ \left(\lambda_{k-n+p}, \rho_{k-n+p}\right) & \text { if } n-p+2 \leq k \leq n\end{cases}
$$

we have $\sum_{k=1}^{n} \tilde{\lambda}_{k} \mu\left(\tilde{\rho}_{k}\right)=0$. Moreover supp $\tilde{\rho}_{1}<\cdots<\operatorname{supp} \tilde{\rho}_{n}$; we are thus led to the previous case and the conclusion follows.

Funny Corollary 4.5. Suppose $\mu$ is a $T_{\nu}$-measure and let $\rho$ be a non-negative $\mu$-integrable function whose support is not negligible. Then $\mu(\rho)$ is non-zero. In particular, $\mu(E)$ is non-zero whenever $\nu(E)$ is non-zero.

Proof. We apply Corollary 4.4 with $p=1, \lambda_{1}=1$.

Remark. This assertion sounds trivial; however the point is that $\mu$ is a vector measure whose components are scalar signed measures. This makes life more difficult. Instead, in the case of oriented measures, this fact is a direct consequence of the definition (since $\mu_{1}$ is then positive). A consequence of the funny corollary is that if $\mu$ is a $T_{\nu}$-measure, then $\nu$ is absolutely continuous with respect to the total variation of $\mu$ !

Lemma 4.6 (perturbation lemma). Suppose $\mu$ is a $T_{\nu}$-measure and let $A_{0}<A_{1}<$ $\cdots<A_{n}$ be $n+1$ measurable sets. Given a positive $\varepsilon$, there exist $n+1$ real numbers $\lambda_{0}, \cdots, \lambda_{n}$ such that

$$
\forall l \in\{0, \cdots, n\} \quad 0<\lambda_{l}<\varepsilon \quad \text { and } \quad \sum_{l=0}^{n}(-1)^{l} \lambda_{l} \mu\left(A_{l}\right)=0 .
$$


Proof. Consider the $n \times n$ linear system

$$
\lambda_{0} \mu\left(A_{0}\right)-\lambda_{1} \mu\left(A_{1}\right)+\cdots+(-1)^{n-1} \lambda_{n-1} \mu\left(A_{n-1}\right)=(-1)^{n-1} \lambda_{n} \mu\left(A_{n}\right),
$$

where $\lambda_{n}$ is a parameter. The determinant of the system is

$$
\omega_{n}=(-1)^{\frac{n(n-1)}{2}} \operatorname{det}\left[\mu\left(A_{0}\right), \cdots, \mu\left(A_{n-1}\right)\right] .
$$

Since $\mu$ is a $T_{\nu}$-measure, $\omega_{n}$ is not zero. For each $i$ in $\{0, \cdots, n-1\}$, let $\omega_{i}$ be

$$
\begin{aligned}
& \qquad \begin{array}{ccccccc}
\mu_{1}\left(A_{0}\right) & \cdots & (-1)^{i-2} \mu_{1}\left(A_{i-2}\right) & (-1)^{n-1} \mu_{1}\left(A_{n}\right) & (-1)^{i} \mu_{1}\left(A_{i}\right) & \cdots & (-1)^{n-1} \mu_{1}\left(A_{n-1}\right) \\
\mu_{2}\left(A_{0}\right) & \cdots & (-1)^{i-2} \mu_{2}\left(A_{i-2}\right) & (-1)^{n-1} \mu_{2}\left(A_{n}\right) & (-1)^{i} \mu_{2}\left(A_{i}\right) & \cdots & (-1)^{n-1} \mu_{2}\left(A_{n-1}\right) \\
\vdots & \ddots & \vdots & \vdots & \vdots & \ddots & \vdots \\
\mu_{n}\left(A_{0}\right) & \cdots & (-1)^{i-2} \mu_{n}\left(A_{i-2}\right) & (-1)^{n-1} \mu_{n}\left(A_{n}\right) & (-1)^{i} \mu_{n}\left(A_{i}\right) & \cdots & (-1)^{n-1} \mu_{n}\left(A_{n-1}\right)
\end{array} \mid ; \\
& \text { i.e. } \quad \omega_{i}=(-1)^{\frac{n(n-1)}{2}} \operatorname{det}\left[\mu\left(A_{0}\right), \cdots, \mu\left(A_{i-2}\right), \mu\left(A_{i}\right), \cdots, \mu\left(A_{n}\right)\right] .
\end{aligned}
$$

By Cramer's formula, $\lambda_{i}$ equals $\lambda_{n} \omega_{i} / \omega_{n}$. The measure $\mu$ being a $T_{\nu}$-measure, $\omega_{i}$ and $\omega_{n}$ have the same sign so that $\lambda_{i}$ is positive whenever $\lambda_{n}$ is positive. Choosing $\lambda_{n}$ such that $0<\lambda_{n}<\min \left(\varepsilon \omega_{n} / \omega_{0}, \cdots, \varepsilon \omega_{n} / \omega_{n-1}, \varepsilon\right)$ we obtain an $(n+1)$-tuple which solves the problem.

\section{The RePresentation theOrem}

We are about to state the main result which allows us to represent the values of a $T_{\nu}$-measure through canonical sets, that we define now.

Notation (canonical sets). The set $\Gamma$ is the subset of $[0,1]^{n}$ defined by

$$
\Gamma=\left\{\left(\gamma_{1}, \cdots, \gamma_{n}\right) \in \mathbb{R}^{n}: 0 \leq \gamma_{1} \leq \cdots \leq \gamma_{n} \leq 1\right\} .
$$

The interior of $\Gamma$ is int $\Gamma=\left\{\left(\gamma_{1}, \cdots, \gamma_{n}\right) \in \mathbb{R}^{n}: 0<\gamma_{1}<\cdots<\gamma_{n}<1\right\}$. For $\gamma=\left(\gamma_{1}, \cdots, \gamma_{n}\right)$ in $\Gamma$, we use the convention $\gamma_{0}=0, \gamma_{n+1}=1$. For each $\gamma=$ $\left(\gamma_{1}, \cdots, \gamma_{n}\right)$ in $\Gamma$, we set

$$
E_{\gamma}=\bigcup_{\substack{0 \leq i \leq n \\ i \text { odd }}}\left(M_{\gamma_{i+1}} \backslash M_{\gamma_{i}}\right)
$$

Theorem 5.1 below was obtained in [3], [4] in the case where $\mu$ is defined on $[0,1]$ and $\nu$ is the Lebesgue measure under some stronger assumptions: in [4], for classical $T$-systems, $\mu$ is assumed to have a continuous density with respect to $\nu$ whereas in [3] $\mu$ satisfies $n$ determinant conditions (instead of one).

Theorem 5.1 (representation theorem). Suppose $\mu$ is a $T_{\nu}$-measure and let $\rho$ be a measurable function such that $0<\rho<1 \nu$-a.e. There exist unique $\alpha$ and $\beta$ in $\Gamma$ satisfying

$$
\mu\left(E_{\alpha}\right)=\int_{X} \rho d \mu=\mu\left(X \backslash E_{\beta}\right) .
$$

Moreover these $\alpha$ and $\beta$ belong to the interior of $\Gamma$.

Remark. The set $X \backslash E_{\beta}$ is dual to the set $E_{\beta}$. In fact

$$
X \backslash E_{\beta}=\bigcup_{\substack{0 \leq i \leq n \\ i \text { even }}}\left(M_{\beta_{i+1}} \backslash M_{\beta_{i}}\right) .
$$


Proof of Theorem 5.1. Assume that the claim concerning the existence and uniqueness of the set $E_{\alpha}$ has been proved. We apply it to the function $1-\rho$. This yields a set $E_{\beta}$ such that $\mu\left(E_{\beta}\right)=\mu(1-\rho)$, which may be rewritten as $\mu\left(X \backslash E_{\beta}\right)=\mu(\rho)$. We will thus only deal with the sets of the form $E_{\alpha}$.

Strict inequalities. Let $\alpha$ be a point of $\Gamma$ such that $\mu\left(E_{\alpha}\right)=\mu(\rho)$. We show that $\alpha$ belongs to the interior of $\Gamma$. We rewrite $\mu\left(E_{\alpha}\right)=\mu(\rho)$ as

$$
\sum_{\substack{0 \leq i \leq n \\ i \text { odd }}} \int_{M_{\alpha_{i+1}} \backslash M_{\alpha_{i}}}(1-\rho) d \mu-\sum_{\substack{0 \leq i \leq n \\ i \text { even }}} \int_{M_{\alpha_{i+1}} \backslash M_{\alpha_{i}}} \rho d \mu=0 .
$$

If we set

$$
\lambda_{i}=(-1)^{i+1}, \quad \rho_{i}= \begin{cases}\rho \chi_{M_{\alpha_{i+1}} \backslash M_{\alpha_{i}}} & \text { if } i \text { is even, } \\ (1-\rho) \chi_{M_{\alpha_{i+1}} \backslash M_{\alpha_{i}}} & \text { if } i \text { is odd }\end{cases}
$$

the equation becomes

$$
\lambda_{0} \int_{X} \rho_{0} d \mu+\cdots+\lambda_{n} \int_{X} \rho_{n} d \mu=0
$$

Let $\mathcal{I}=\left\{i: 0 \leq i \leq n, \alpha_{i}<\alpha_{i+1}\right\}$; note that for $i$ in $\mathcal{I}$ the function $\rho_{i}$ is strictly positive on $M_{\alpha_{i+1}} \backslash M_{\alpha_{i}}$. Assume that the $n$-tuple $\alpha$ does not belong to the interior of $\Gamma$. Then $|\mathcal{I}| \leq n$; if we write $\mathcal{I}=\left\{i_{1}, \cdots, i_{p}\right\}$, where $i_{1}<\cdots<i_{p}$, we have

$$
1 \leq p \leq n, \quad \sum_{j=1}^{p} \lambda_{i_{j}} \int_{X} \rho_{i_{j}} d \mu=0, \quad \operatorname{supp} \rho_{i_{1}}<\cdots<\operatorname{supp} \rho_{i_{p}}, \lambda_{i_{j}} \in\{-1,1\} .
$$

Corollary 4.4 yields a contradiction. It follows that $0<\alpha_{1}<\cdots<\alpha_{n}<1$.

Uniqueness. Let $\delta=\left(\delta_{1}, \cdots, \delta_{n}\right)$ and $\gamma=\left(\gamma_{1}, \cdots, \gamma_{n}\right)$ be two elements of $\Gamma$ such that $\mu\left(E_{\delta}\right)=\mu(\rho)=\mu\left(E_{\gamma}\right)$. The first part of the proof (strict inequalities) shows that $0<\delta_{1}<\cdots<\delta_{n}<1$ and $0<\gamma_{1}<\cdots<\gamma_{n}<1$. Assume for instance that $\delta_{1} \leq \gamma_{1}$; then $E_{\delta} \cap M_{\delta_{1}}=E_{\gamma} \cap M_{\delta_{1}}=\emptyset$ so that the equality $\mu\left(E_{\delta}\right)=\mu\left(E_{\gamma}\right)$ becomes

$$
\int_{X \backslash M_{\delta_{1}}}\left(\chi_{E_{\delta}}-\chi_{E_{\gamma}}\right) d \mu=0 .
$$

The sets $\left(M_{\delta_{i+1}} \backslash M_{\delta_{i}}\right)_{1 \leq i \leq n}$ cover $X \backslash M_{\delta_{1}}$; moreover on $M_{\delta_{i+1}} \backslash M_{\delta_{i}}$ we recall that $\chi_{E_{\delta}}=1$ if $i$ is odd and $\chi_{E_{\delta}}=0$ if $i$ is even. The above equality then yields

$$
\sum_{\substack{0 \leq i \leq n \\ i \text { odd }}} \int_{M_{\delta_{i+1}} \backslash M_{\delta_{i}}}\left(1-\chi_{E_{\gamma}}\right) d \mu-\sum_{\substack{2 \leq i \leq n \\ i \text { even }}} \int_{M_{\delta_{i+1}} \backslash M_{\delta_{i}}} \chi_{E_{\gamma}} d \mu=0
$$

which may be rewritten

$$
\sum_{i=1}^{n} \lambda_{i} \int_{X} \rho_{i} d \mu=0 \quad \text { where } \quad \lambda_{i}=(-1)^{i+1}, \quad \rho_{i}=\left|\chi_{E_{\delta}}-\chi_{E_{\gamma}}\right| \chi_{M_{\delta_{i+1}} \backslash M_{\delta_{i}}} .
$$


Now each $\rho_{i}$ is non-negative. If $\delta \neq \gamma$ there exists $j$ such that $\rho_{j}$ is positive on a non-negligible set. By Corollary $4.5, \mu\left(\rho_{j}\right)$ is non-zero so that the set $\mathcal{J}=\{i$ : $\left.\mu\left(\rho_{i}\right) \neq 0\right\}$ is not empty. Writing $\mathcal{J}=\left\{i_{1}, \cdots, i_{p}\right\}$, where $i_{1}<\cdots<i_{p}$, we have

$$
1 \leq p \leq n, \quad \sum_{k=1}^{p} \lambda_{i_{k}} \int_{X} \rho_{i_{k}} d \mu=0, \quad \operatorname{supp} \rho_{i_{1}}<\cdots<\operatorname{supp} \rho_{i_{p}}, \lambda_{i_{k}} \in\{-1,1\} .
$$

Corollary 4.4 yields a contradiction. It follows that $\delta=\gamma$.

Existence. Let $\theta: \Gamma \rightarrow \mathbb{R}^{n}$ be the map defined by $\theta(\alpha)=\mu\left(E_{\alpha}\right)$. The nonatomicity of $\mu$ with respect to the $M_{i}$ 's implies that this map is continuous. Moreover the second part of the proof (uniqueness) shows that the map $\theta$ is injective on int $\Gamma$. The invariance domain theorem [6] then implies that $\theta($ int $\Gamma)$, the image of int $\Gamma$, is open in $\mathbb{R}^{n}$.

Surprisingly $\theta($ int $\Gamma)$ is contained in the convex set

$$
C=\left\{\int_{X} \rho d \mu: 0<\rho<1 \quad \nu \text { - a.e. }\right\} .
$$

In fact, let $\alpha=\left(\alpha_{1}, \cdots, \alpha_{n}\right)$ belong to int $\Gamma$. Applying the perturbation lemma, Lemma 4.6 , to $\mu, A_{i}=M_{\alpha_{i+1}} \backslash M_{\alpha_{i}}$ and $\varepsilon=1 / 4$ we obtain a $(n+1)$-tuple $\left(\lambda_{0}, \cdots, \lambda_{n}\right)$ such that

$$
\forall i \in\{0, \cdots, n\} \quad 0<\lambda_{i}<1 / 4 \text { and } \sum_{i=0}^{n}(-1)^{i} \lambda_{i} \mu\left(A_{i}\right)=0 .
$$

Put

$$
\rho=\sum_{\substack{0 \leq i \leq n \\ i \text { even }}} \lambda_{i} \chi_{A_{i}}+\sum_{\substack{0 \leq i \leq n \\ i \text { odd }}}\left(1-\lambda_{i}\right) \chi_{A_{i}} .
$$

By construction we have $0<\rho<1$ and $\mu(\rho)=\theta(\alpha)$ so that $\theta(\alpha)$ belongs to $C$.

Now the first part of the proof (strict inequalities) shows that $\theta(\Gamma) \cap C=\theta($ int $\Gamma)$; the compactness of $\Gamma$ then implies that $\theta(\operatorname{int} \Gamma)$ is closed in $C$. The convex set $C$ is connected; $\theta(\operatorname{int} \Gamma$ ) is open and closed in $C$. Thus it coincides with the whole set $C$.

Remark. The map $\theta$ was first introduced in [2] to prove Theorem 5.1 under the stronger assumptions that $\mu$ is defined on $[0,1]$ and admits a continuous density with respect to the Lebesgue measure. In [2] however $\theta$ is differentiable and a local homeomorphism: Caccioppoli's global inversion theorem yields the injectivity of $\theta$ on int $\Gamma$ and the fact that $\theta$ (int $\Gamma$ ) is open; instead here we first prove directly the injectivity of $\theta$ (without being differentiable) and then we apply the open mapping theorem.

A simple classical approximation argument yields the following corollary.

Corollary 5.2. Suppose $\mu$ is a $T_{\nu}$-measure. Let $\rho$ be a measurable function such that $0 \leq \rho \leq 1$. There exist $\alpha$ and $\beta$ in $\Gamma$ satisfying

$$
\mu\left(E_{\alpha}\right)=\int_{X} \rho d \mu=\mu\left(X \backslash E_{\beta}\right) .
$$

We denote by $\mathcal{R}$ the range of $\mu$; i.e. $\mathcal{R}=\{\mu(E): E \in \mathcal{A}\}$. 
Remark. The proof of Theorem 5.1 shows that $\theta$ (int $\Gamma$ ) is open and convex; by Corollary 5.2 its closure coincides with $\mathcal{R}$. Then by $[7$, Th. 6.3$]$ we obtain that int $\mathcal{R}=\theta($ int $\Gamma)$.

Corollary 5.3. Suppose $\mu$ is a $T_{\nu}$-measure. Let $\rho$ be a measurable function such that $0 \leq \rho \leq 1$ and $0<\rho<1$ on a $\nu$-non negligible set. Then $\mu(\rho)$ belongs to the interior of $\mathcal{R}$; in particular there exist unique $\alpha$ and $\beta$ in the interior of $\Gamma$ satisfying

$$
\mu\left(E_{\alpha}\right)=\int_{X} \rho d \mu=\mu\left(X \backslash E_{\beta}\right) .
$$

Proof. There exist a $\nu$-non negligible set $F$ and $\varepsilon>0$ such that $\varepsilon \leq \rho \leq 1-\varepsilon$ on $F$. The non-atomicity assumption yields the existence of $0<\delta_{1}<\cdots<\delta_{n}<1=\delta_{n+1}$ such that if for every $i$ we set $A_{i}=\left(M_{\delta_{i+1}} \backslash M_{\delta_{i}}\right) \cap F$ then $\nu\left(A_{i}\right)>0$. Therefore $A_{1}<\cdots<A_{n}$ and the vectors $\mu\left(A_{1}\right), \cdots, \mu\left(A_{n}\right)$ are linearly independent. It follows that the open set $V=\left\{\sum_{i=1}^{n} \lambda_{i} \mu\left(A_{i}\right):\left|\lambda_{i}\right|<\varepsilon, \quad i=1, \cdots, n\right\}$ is a neighborhood of $O$ in $\mathbb{R}^{n}$. Now for every $\lambda_{1}, \cdots, \lambda_{n}$ such that $\left|\lambda_{i}\right|<\varepsilon$ we have $0 \leq \rho+\sum_{i=1}^{n} \lambda_{i} \chi_{A_{i}} \leq 1$ a.e. on $X$; it follows that the neighborhood $\mu(\rho)+V$ of $\mu(\rho)$ in $\mathbb{R}^{n}$ is contained in $\mathcal{R}$.

The following results have been stated in a less general context in [3] but they are still valid in the present framework; Chebyshev measures provide a broad class of measures whose range is strictly convex.

Theorem 5.4. The range $\mathcal{R}$ of a $T_{\nu}$-measure is strictly convex. The boundary points of $\mathcal{R}$ admit a unique representation modulo $\mu$. Moreover a point $\mu(E)$ belongs to the boundary of $\mathcal{R}$ if and only if there exists $\gamma$ in the boundary of $\Gamma$ such that $\mu\left(E \Delta E_{\gamma}\right)=0$.

Finally, we remark that the same results would hold under a weaker assumption on the measure. Namely, it is enough that for each $n$-tuple of measurable sets $A_{1}, \cdots, A_{n}$ such that $A_{1}<\cdots<A_{n}$, the $\operatorname{determinant} \operatorname{det}\left[\mu\left(A_{1}\right), \cdots, \mu\left(A_{n}\right)\right]$ is positive. The delicate point concerns Theorem 4.2 which is the key for proving the representation theorem. In this situation, the proof should be done along the lines of Theorem 2.2 of [3].

\section{REFERENCES}

1. R. Cerf - C. Mariconda, On bang-bang constrained solutions of a control system, SIAM J. Control Optim. 33 (1995), 554-567. MR 95m:49019

2. R. Cerf - C. Mariconda, Oriented measures with continuous densities and the bang-bang principle, J. Funct. Anal. 126 (1994), 476-505. MR 95k:49015

3. R. Cerf - C. Mariconda, Oriented measures, J. Math. Anal. Appl. 197 (1996), 925-944. CMP 96:07

4. S. Karlin - W.J. Studden, Tchebycheff systems: with applications in analysis and statistics, Pure and applied mathematics, John Wiley \& Sons, New York, 1966. MR 34:4757

5. M.G. KreĬn - A.A. Nudel'man, The Markov moment problems and extremal problems, Transl. Math. Monographs, vol. 50, AMS, Providence, Rhode Island, 1977. MR 56:16284

6. N.G. Lloyd, Degree theory, Cambridge University Press, Cambridge, 1978. MR 58:12558

7. R.T. Rockafeller, Convex Analysis, Princeton University Press, Princeton, New Jersey, 1972.

Université Paris sud, Mathématiques, bâtiment 425, 91405 Orsay Cedex, France

E-mail address: Raphael.Cerf@math.u-psud.fr

Dipartimento di Matematica pura e applicata, Università di Padova, 7 via Belzoni, 35131 Padova, Italy

E-mail address: mariconda@math.unipd.it 